UCRHEP-T394

August 2005

\title{
Efficacious Additions to the Standard Model
}

\author{
Ernest Ma \\ Physics Department, University of California, Riverside, California 92521, USA, \\ and Institute for Particle Physics Phenomenology, Department of Physics, \\ University of Durham, Durham, DH1 3LE, UK
}

\begin{abstract}
If split supersymmetry can be advocated as a means to have gauge-coupling unification as well as dark matter, another plausible scenario is to enlarge judiciously the particle content of the Standard Model to achieve the same goals without supersymmetry. A simple efficacious example is presented.
\end{abstract}


It has been proposed in the last year that supersymmetry could be split [1] so that scalar quarks and leptons are very heavy whereas gauginos and higgsinos remain light at the electroweak scale. This scenario ignores the hierarchy problem but has the virtue of retaining gauge-coupling unification as well as dark matter. It has inspired a large number of phenomenological studies. On the other hand, if the goal is simply to preserve gaugecoupling unification and the appearance of dark matter, then a judicious choice of particle content beyond that of the Standard Model (SM) works just as well. Such an alternative may have very different predictions at the $\mathrm{TeV}$ scale and should not be overlooked in this context. A simple efficacious example is presented below.

It has been known for a long time that the particle content of the SM does not lead to unification of the three gauge couplings of the standard $S U(3)_{C} \times S U(2)_{L} \times U(1)_{Y}$ gauge group, whereas that of the Minimal Supersymmetric Standard Model (MSSM) does [2]. To understand how this works, consider the one-loop renormalization-group equations governing the evolution of these couplings with mass scale:

$$
\frac{1}{\alpha_{i}\left(M_{1}\right)}-\frac{1}{\alpha_{i}\left(M_{2}\right)}=\frac{b_{i}}{2 \pi} \ln \frac{M_{2}}{M_{1}}
$$

where $\alpha_{i}=g_{i}^{2} / 4 \pi$ and the numbers $b_{i}$ are determined by the particle content of the model between $M_{1}$ and $M_{2}$. In the SM with one Higgs scalar doublet, these are given by

$$
\begin{aligned}
& S U(3)_{C}: \quad b_{C}=-11+(4 / 3) N_{f}=-7, \\
& S U(2)_{L}: \quad b_{L}=-22 / 3+(4 / 3) N_{f}+1 / 6=-19 / 6, \\
& U(1)_{Y} \quad: \quad b_{Y}=(4 / 3) N_{f}+1 / 10=41 / 10,
\end{aligned}
$$

where $N_{f}=3$ is the number of quark and lepton families and $b_{Y}$ has been normalized by the well-known factor of $3 / 5$. In the MSSM with two Higgs superfields, the shifts in $b_{i}$ from those of the SM are given by

$$
\Delta b_{C}=(2 / 3) N_{f}+2,
$$




$$
\begin{aligned}
\Delta b_{L} & =(2 / 3) N_{f}+13 / 6 \\
\Delta b_{Y} & =(2 / 3) N_{f}+1 / 2
\end{aligned}
$$

Since the scalar quarks and leptons are contained in the terms proportional to $N_{f}$, they do not change the relative values of $b_{i}$. Hence they do not affect the unification condition

$$
\alpha_{C}\left(M_{U}\right)=\alpha_{L}\left(M_{U}\right)=(5 / 3) \alpha_{Y}\left(M_{U}\right)=\alpha_{U}
$$

or the value of $M_{U}$; they only change the value of $\alpha_{U}$. Split supersymmetry is essentially the scenario where

$$
\Delta b_{C}=2, \quad \Delta b_{L}=13 / 6, \quad \Delta b_{Y}=1 / 2,
$$

which is realized by adding to the SM new fermions transforming as $(8,1,0),(1,3,0),(1,1,0)$, $(1,2, \pm 1 / 2)$, and a second scalar Higgs doublet. Suppose instead we add two complex scalar octets $\zeta, \zeta^{\prime} \sim(8,1,0)$, one Majorana fermion triplet $f \sim(1,3,0)$ as well as one complex scalar triplet $s \sim(1,3,0)$, then

$$
\Delta b_{C}=2, \quad \Delta b_{L}=2, \quad \Delta b_{Y}=0
$$

which mimics Eq. (9) to a large extent. Assuming that these new particles appear at around $M_{X}$, then the unification condition of Eq. (8) implies

$$
\begin{aligned}
\frac{1}{\alpha_{C}\left(M_{Z}\right)} & =\frac{3}{158}\left[\frac{91}{\alpha_{L}\left(M_{Z}\right)}-\frac{23}{\alpha_{Y}\left(M_{Z}\right)}\right]+\frac{15}{158 \pi} \ln \frac{M_{X}}{M_{Z}}, \\
\ln \frac{M_{U}}{M_{Z}} & =\frac{30 \pi}{79}\left[\frac{3}{5 \alpha_{Y}\left(M_{Z}\right)}-\frac{1}{\alpha_{L}\left(M_{Z}\right)}\right]-\frac{30}{79} \ln \frac{M_{X}}{M_{Z}} .
\end{aligned}
$$

Using the input 3

$$
\begin{aligned}
& \alpha_{L}\left(M_{Z}\right)=(\sqrt{2} / \pi) G_{F} M_{W}^{2}=0.0340 \\
& \alpha_{Y}\left(M_{Z}\right)=\alpha_{L}\left(M_{Z}\right) \tan ^{2} \theta_{W}=0.0102,
\end{aligned}
$$

and

$$
0.115<\alpha_{C}\left(M_{Z}\right)<0.119
$$


we find

$$
1800 \mathrm{GeV}>M_{X}>500 \mathrm{GeV},
$$

and

$$
5.1 \times 10^{16} \mathrm{GeV}<M_{U}<8.3 \times 10^{16} \mathrm{GeV}
$$

These are certainly acceptable values for new particles at the $\mathrm{TeV}$ scale and the proper suppression of proton decay.

The new particles assumed are adjoint representations of $S U(3)_{C}$ or $S U(2)_{L}$. They could for example be components of the adjoint $\underline{24}$ representation of $S U(5)$. There is no fundamental understanding of why they are light, but the other components are heavy, just as there is no fundamental understanding of why the scalar $S U(2)_{L}$ doublet components of the $\underline{5}$ representation of $S U(5)$ are light, but the $S U(3)_{C}$ triplet components are heavy in the canonical $S U(5)$ model of grand unification.

We now come to the phenomenology of the new particles. The $S U(3)_{C}$ scalar octets $\zeta, \zeta^{\prime}$ may be thought of as scalar gluons. They decay into two vector gluons in one loop, i.e. $\zeta \rightarrow \zeta \zeta \rightarrow g g$, etc. If kinematically allowed, they would be produced copiously at the Large Hadron Collider (LHC) and easily detected. As far as $\Delta b_{C}=2$ in Eq. (10) is concerned, $\eta$ and $\zeta$ may be replaced by a single Majorana fermion octet, i.e. the gluino in the MSSM, but then the latter would be absolutely stable without scalar quarks, which is not acceptable cosmologically. [In split supersymmetry, the scalar quarks are still present, only much heavier.]

Instead of the required two Higgs superfields in the MSSM, this model has only the single Higgs boson of the SM. Hence its mass is not constrained by the MSSM and may exceed the latter's current upper bound of about $127 \mathrm{GeV}$ 4].

The Majorana fermion triplet $\left(f^{+}, f^{0}, f^{-}\right)$are like the three $S U(2)_{L}$ gauginos of the 
MSSM. However, there is no $U(1)_{Y}$ gaugino here. After all, it contributes nothing to $b_{C}$, $b_{L}$, or $b_{Y}$, so it is not necessary for gauge-coupling unification. In the MSSM, the gauginos couple to the Higgs scalars through the higgsinos, but here there are no higgsinos. However, since the left-handed leptons $(\nu, l)$ are doublets, $f$ can couple to them through the SM Higgs doublet, which would allow $f$ to decay and disqualify it from being considered as dark matter. The solution here is the same as in the MSSM. We simply assign a conserved quantity to $f$, say a multiplicative parity under which $f$ is odd and all other particles are even, in exact analogy to $R$-parity in the MSSM.

The complex scalar triplet $\left(s^{+}, s^{0}, s^{-}\right)$couples to the SM Higgs doublet $\left(\phi^{+}, \phi^{0}\right)$ according to

$$
s^{+} \phi^{-} \phi^{0}+s^{0}\left(\bar{\phi}^{0} \phi^{0}-\phi^{-} \phi^{+}\right) / \sqrt{2}-s^{-} \bar{\phi}^{0} \phi^{+},
$$

which means that $s^{0}$ will acquire a nonzero vacuum expectation value. This has to be smaller than about a $\mathrm{GeV}$ to satisfy the precision electroweak measurements of the $W$ and $Z$ masses. It is nevertheless important for the phenomenology of dark matter because it allows $f^{ \pm}$to be heavier than $f^{0}$ from the coupling

$$
s^{+} f^{0} f^{-}+s^{0} f^{-} f^{+}+s^{-} f^{+} f^{0},
$$

so that $f^{ \pm}$may decay weakly into $f^{0}$ plus a virtual $W^{ \pm}$which becomes a quark-antiquark pair or lepton-antilepton pair. Thus $f^{0}$ (the analog of the neutral wino in the MSSM) is the candidate for dark matter in this model.

The idea that particles beyond those of the SM can restore gauge-coupling unification and suppress proton decay is not new [5], but with the possible abandonment of low-energy supersymmetry as the only acceptable scenario below the TeV scale, new alternatives should be explored. One such simple example has been presented in this paper, with experimentally verifiable predictions very different from those of the MSSM or split supersymmetry. 
This work was supported in part by the U. S. Department of Energy under Grant No. DE-FG03-94ER40837.

\section{References}

[1] N. Arkani-Hamed and S. Dimopoulos, hep-th/0405159 G. F. Giudice and A. Romanino, Nucl. Phys. B699, 65 (2004) [Erratum: ibid. B706, 65 (2005)]; N. Arkani-Hamed, S. Dimopoulos, G. F. Giudice, and A. Romanino, ibid. B709, 3 (2005). See also J. D. Wells, Phys. Rev. D71, 015013 (2005).

[2] See for example W. de Boer and C. Sander, Phys. Lett. B585, 276 (2004).

[3] S. Eidelman et al., Particle Data Group, Phys. Lett. B592, 1 (2004).

[4] See for example R. Kinnunen et al., J. Phys. G31, 71 (2005).

[5] See for example K. S. Babu and E. Ma, Phys. Lett. 144B, 381 (1984); also W. Grimus and L. Lavoura, Eur. Phys. J. C28, 123 (2003). 